\title{
Toward Seamless Wearable Sensing: Automatic On-Body Sensor Localization for Physical Activity Monitoring
}

\author{
Ramyar Saeedi ${ }^{1}$, Janet Purath ${ }^{2}$, Krishna Venkatasubramanian ${ }^{3}$, and Hassan Ghasemzadeh ${ }^{1}$
}

\begin{abstract}
Mobile wearable sensors have demonstrated great potential in a broad range of applications in healthcare and wellness. These technologies are known for their potential to revolutionize the way next generation medical services are supplied and consumed by providing more effective interventions, improving health outcomes, and substantially reducing healthcare costs. Despite these potentials, utilization of these sensor devices is currently limited to lab settings and in highly controlled clinical trials. A major obstacle in widespread utilization of these systems is that the sensors need to be used in predefined locations on the body in order to provide accurate outcomes such as type of physical activity performed by the user. This has reduced users' willingness to utilize such technologies. In this paper, we propose a novel signal processing approach that leverages feature selection algorithms for accurate and automatic localization of wearable sensors. Our results based on real data collected using wearable motion sensors demonstrate that the proposed approach can perform sensor localization with $98.4 \%$ accuracy which is $30.7 \%$ more accurate than an approach without a feature selection mechanism. Furthermore, utilizing our node localization algorithm aids the activity recognition algorithm to achieve $98.8 \%$ accuracy (an increase from $33.6 \%$ for the system without node localization).
\end{abstract}

\section{INTRODUCTION}

Physical activity is an important component of many chronic conditions such as diabetes, heart failure, cardiovascular disease, and cancer. Physical activity is defined as bodily movement produced by the contraction of skeletal muscle to substantially increase energy expenditure. Participation in regular physical activity improves blood glucose control and can prevent or delay diabetes and heart diseases. Observed benefits of physical activity include positive effects on lipids, blood pressure, cardiovascular events, mortality, and quality of life [1], [2].

Recent advancements in wearable sensors and wireless communication allow continuous and remote monitoring of physical activities in patients with chronic conditions. In particular, wearable motion sensors such as accelerometers and gyroscopes [3] with embedded wireless connectivity are used for physical activity monitoring. Physical activity interventions using wearable sensors often involve activity recognition, which refers to detection of activity types

\footnotetext{
${ }^{1}$ R. Saeedi and H. Ghasemzadeh are with the School of Electrical Engineering and Computer Science, Washington State University, Pullman, WA 99164 USA (phone: 509-335-8260; e-mail: \{rsaeedi, hassan\}@eecs.wsu.edu).

${ }^{2}$ J. Purath is with the College of Nursing, Washington State University, Spokane, WA 99210 USA (e-mail: jpurath@wsu.edu)

${ }^{3} \mathrm{~K}$. Venkatasubramanian is with the Computer Science Department, Worcester Polytechnic Institute, Massachusetts, MA 01609 USA (e-mail: kven@wpi.edu).
}

(e.g., walking, running, sitting, biking, and standing). Unfortunately, current approaches for activity recognition use predefined sensor locations, which impose much discomfort for patients as they are not allowed to use sensors on their own desired body locations. For example, while one patient may prefer to use his/her smartphone in his pocket, another patient may prefer to carry the smartphone in a backpack or purse. One patient may prefer to use a wrist-band sensor on his right wrist, while another patient may prefer a left wrist setting. Failure to adhere to the predefined protocols (i.e., using sensors of pre-specified body locations) results in drastic reduction in accuracy of physical activity monitoring such as activity recognition algorithms. Therefore, new algorithms and signal processing techniques are needed to detect location of wearable sensor automatically and in real-time as they are being used by patients. This problem is generally referred to as on-body sensor localization.

\section{A. Seamless Wearable Sensing}

An important aspect of designing future mobile wearable sensors is to offer these systems in a plug-and-play manner. In other words, end users need to handle different types of sensors with a minimum amount of supervision and intervention. Since elderly and non-expert individuals are primary users of these systems, reliability, ease of use, and robustness tend to be main challenges of large scale mobile wearable sensors. Consequently, an urgent need is to design these systems in a way that they deliver non-intrusive, seamless and automatically configurable sensing. Automatic node localization is an important aspect of seamless sensing. By performing node localization automatically and accurately, end users can use the system with no concerns on how/where the sensor node needs to be worn on the body. As a result, automatic on-body localization is needed to enhance usability, adaptability, ease of setup, and accuracy of mobile wearable sensors.

\section{B. Related Work}

The ultimate goal of on-body node localization is to find the location of the wearable sensor nodes prior to further processing the motion sensor signals (e.g., accelerometers, gyroscopes). Activity recognition is one example of the physical activity monitoring whose performance can be severely compromised in absence of an automatic node localization algorithm. The number and location of wearable sensors vary in different activity recognition applications. A survey study in [3] reports that the number of sensor nodes used for activity recognition may vary from a single node to 19 sensor 
nodes resulting in an accuracy that ranges from $79 \%$ to $98 \%$. The most common algorithms for movement classification using wearable motion sensors include k-Nearest-Neighbor $(\mathrm{kNN})$, Hidden Markov Models (HMM) and Support Vector Machine (SVM) [4]. Based on these specifications, we use a typical activity recognition framework as a basis for on-body sensor localization. Therefore, we minimize the overhead of such algorithms to the system.

Most activity recognition algorithms use a fix set of nodes and predetermined placement. However, there has been less effort in detecting location of wearable nodes. Major drawbacks with current on-body sensor localization algorithms include either lack of sufficient accuracy or need for a priori knowledge about the activity being performed by the subject. For example, a recent study in [5] developed an on-body sensor localization algorithm that applies only to walking movements. The approach presented in [6] assumes that movement types are known a priori. In real world applications, however, we are often facing situations that knowledge about human movements does not exist a priori. In fact, activity recognition is often the main goal of utilizing mobile wearable motion sensors.

Authors in [7] proposed an algorithm for on-body position of wearable sensors. They introduced a method to determine the time period of walking, and then they used a $\mathrm{C} 4.5$ classifier to find the location of node in four different places (wrist, breast pocket, trousers pocket, and right eye). In another study [8], authors introduced a method to perform localization based on daily activity routines. This limited set of nodes is not applicable to many current body sensor networks. Most of applications will need much more number of sensors.

Another localization algorithm is presented in [5]. While this study enhances the number of sensors compare to previous works, implementation of algorithm is based on walking activity. They used an unsupervised technique to discover the walking activity based on frequency and consistency during long period of time. Another paper introduced a method to find that two portable devices are carried by the same person [9].

\section{Contributions}

On-body sensor localization without prior knowledge about the type of the activity being performed is a hard problem mainly due to the large number of potential body locations that can accommodate a wearable sensor and also the large amount of activities that can be performed. Prior research in this area either assumes that the type of activity is known a priori or uses computationally expensive processing algorithms for node localization. Furthermore, research in the area of on-body sensor localization is very new and it is not clear what algorithms are most effective in detecting sensor locations and what factors affect performance of such algorithms. Motivated by these needs, we study the problem of on-body sensor localization in the context of pattern recognition and signal processing. Our goal in this paper is to identify signal processing algorithms that are promising

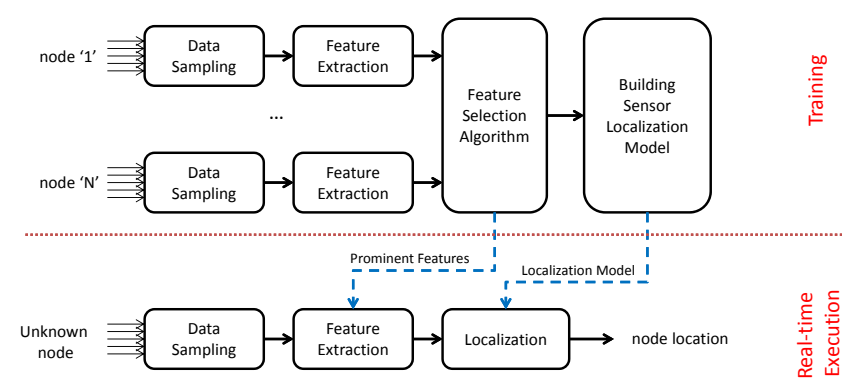

Fig. 1. Overall data processing flow: training phase and real-time execution of the sensor localization algorithm

for on-body sensor localization and determine parameters that affect performance of such algorithms. To this end, we use real data collected from several subjects performing transitional movements to develop and fine tune classification algorithms for sensor location detection. Our results show that a computationally simple classification algorithm such as k-Nearest-Neighbor $(\mathrm{kNN})$ classifier that operates on a small but intelligently constructed feature space can provide over $98.4 \%$ accuracy in detecting 7 wearable sensor nodes used for activity recognition.

\section{Materials And Methods}

Our approach in using acceleration and angular velocity data for on-body localization is motivated by applications of these sensors in activity recognition. Most previous work finds location of the nodes based on a specific activity (e.g., walking). This assumption requires that the users repeat predefined activities before practical use of monitoring system. Such a calibration procedure may create even more burden that using a setting of sensor nodes with predefined on-body locations. As we mentioned previously, the main goal is to provide a non-intrusive way of installation that help us move toward fully plug-and-play wearable sensing. In our approach, we attempt to provide a method to execute the localization with minimum intrusion. Our approach to onbody sensor localization is pattern recognition and event classification, where sensor locations are considered as events of interest to be detected. The first step is to collect data for variety of movements. Next, we extract an exhaustive set of features from the collected acceleration and angular velocity signals. We then select most prominent features from the large set of feature pool. A variety of classification algorithms are then applied on the reduced feature space to assess performance of the sensor localization algorithm. A high level diagram demonstrating training and execution phases of the algorithms is shown in Fig. 1. In the following, details of each step are provided.

\section{A. Data Collection}

We used a network of wearable motion sensors with accelerometer and gyroscope sensors and wireless connectivity to collect data. Fig. 2 shows the body locations on which the sensor is worn during data collection. The data collection was performed for a variety of movements. The reason for using different types of movements is to create a reasonable 


\begin{tabular}{|c|l|}
\hline ID & \multicolumn{1}{|c|}{ Location } \\
\hline 1 & Waist \\
\hline 2 & Right Wrist (R-Wrist) \\
\hline 3 & Left Wrist (L-Wrist) \\
\hline 4 & Right Arm (R-Arm) \\
\hline 5 & Left Thigh (L-Thigh) \\
\hline 6 & Right Ankle (R-Ankle) \\
\hline 7 & Left Ankle (L-Ankle) \\
\hline
\end{tabular}

Fig. 2. Wearable sensor locations

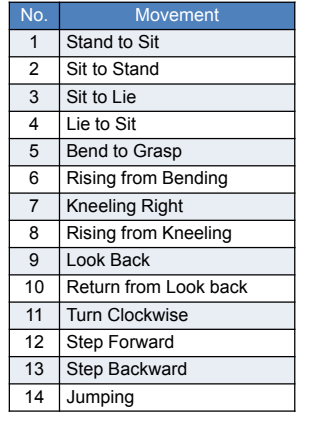

(a) Experimental Movements

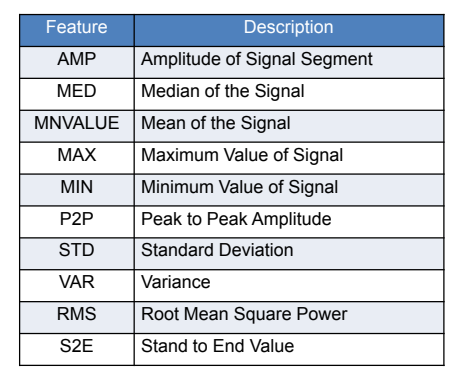

(b) Extracted features from each sensor signal
Fig. 3. List of experimental movements and extracted features

dataset for training a classifier for localization purposes. Therefore, on-body localization could be performed without supervision in the testing mode. The data collection is performed with 14 different types of transitional movements, so the system could be able to detect the patterns in most common activities of daily life. A list of the transitional movements is shown in Fig. 3(a). Three healthy subjects aged between 25 and 35 were asked to perform each experimental movement 10 times. Each sensor nodes was programmed to wirelessly transmit data to a laptop computer where all the data were stored for off-line data processing and algorithm development.

\section{B. Feature Extraction}

Potentially, there are many different features that can be extracted from human activity signals. Previous studies use statistical features that have shown effective in human activity recognition. For node localization purposes, however, it is largely unknown what features are most effective. Thus, one of our goals in this paper was to explore features that are most effective for node localization. For this purpose, we extracted an exhaustive set of features that may be useful for on-body localization. The set of features extracted from individual sensor streams is shown in Fig. 3(b). In addition to these features, we extracted 10 morphological features from each signal segment. Morphological features attempt to capture structural properties of the signal. In this paper, morphological features are samples that are evenly spaced in time over the entire signal segment associated with a particular movement.

\section{Feature Selection}

Each one of the seven sensor nodes in our network consists of a 2-axis gyroscope and a 3-axis accelerometer. Thus,
TABLE I

SELECTED FEATURES

\begin{tabular}{cll}
\hline No. & Feature & Sensor \\
\hline 1 & MED & Y-Axis Gyro \\
2 & MNVALUE & Y-Axis Acc \\
3 & MIN & X-Axis Gyro \\
4 & STD & Z-Axis Acc \\
5 & STD & X-Axis Gyro \\
6 & STD & Y-Axis Gyro \\
\hline
\end{tabular}

the total number of features is relatively high for real-time execution on wearable sensor nodes with limited processing power and energy sources. Furthermore, an exhaustive feature set potentially includes features that can lower accuracy of sensor localization. Therefore, we use a feature selection algorithm to lower the number of signal segment features. We utilize the feature selection algorithm in [10] to select a small subset of the extracted features for sensor localization.

\section{RESULTS}

As shown in Fig. 3(b) and discussed in Section II-B, we extracted 20 features from each signal segment. Given that each sensor node has 5 embedded sensor axes, the total number of features extracted from each sensor node is $20 \times 5=100$. We applied the feature selection algorithm discussed in Section II-C to reduce this number. The results show that only 6 features are prominent for sensor node localization. These features are listed in Table I.

Table II shows accuracy performance of our node localization using a kNN classifier. As it can be observed from this table, all the nodes can be detected with a minimum recall of $94 \%$ and minimum precision of $96 \%$. On average, the system achieves $98.41 \%$ and $98.42 \%$ recall and precision respectively.

Table III shows the accuracy of our sensor localization as well as the compressive sensing approach in [6]. As shown in this table, the feature selection algorithm plays an important role in eliminating insignificant features that can lower the accuracy of the node localization algorithm. When all features (i.e., 100 features per node) are used for localization, the overall accuracy of the $\mathrm{kNN}$ algorithm is only $75.3 \%$. The feature selection algorithm improves the accuracy by $30.7 \%$. Beside the $\mathrm{kNN}$ classifier, three other classifiers are used for comparison. The SVM classifier obtains $99.8 \%$ accuracy which is the highest accuracy among all other cases. Furthermore, while our localization algorithm uses only 6 features from each sensor node, it outperforms the algorithm presented in [6] which uses a large number of features for node localization.

Fig. 4 shows the accuracy of activity recognition in presence of our sensor localization algorithm compared to the case without a sensor localization algorithm. For the case without localization, we assumed that sensor nodes are randomly placed on the body. We repeated the experiment 10 times each with a pseudo random combination of the nodes. When an accurate localization algorithm is utilized, the activity recognition algorithm (a kNN neighbor classifier) achieves $98.8 \%$ accuracy in detecting the 14 movements. 
TABLE II

PERFORMANCE OF THE PROPOSED NODE LOCALIZATION ALGORITHM USING FEATURES IN TABLE I

\begin{tabular}{llllllll|ll}
\hline & Waist & R-Wrist & L-Wrist & R-Arm & L-Thigh & R-Ankle & L-Ankle & Total & Recall \\
\hline Waist & 209 & 0 & 0 & 0 & 1 & 0 & 0 & 210 & $99.5 \%$ \\
R-Wrist & 2 & 208 & 0 & 0 & 0 & 0 & 0 & 210 & $99.0 \%$ \\
L-Wrist & 0 & 0 & 209 & 0 & 1 & 0 & 0 & 210 & $99.5 \%$ \\
R-Arm & 0 & 0 & 0 & 210 & 0 & 0 & 0 & 210 & $100 \%$ \\
L-Thigh & 3 & 0 & 0 & 0 & 199 & 8 & 0 & 210 & $94.7 \%$ \\
R-Ankle & 2 & 0 & 0 & 0 & 6 & 202 & 0 & 210 & $96.2 \%$ \\
L-Ankle & 0 & 0 & 0 & 0 & 0 & 0 & 210 & 210 & $100 \%$ \\
\hline Total & 216 & 208 & 209 & 210 & 207 & 210 & 210 & & \\
Precision & $97 \%$ & $100 \%$ & $100 \%$ & $100 \%$ & $96 \%$ & $96 \%$ & $100 \%$ & & \\
\hline
\end{tabular}

TABLE III

ACCURACY (IN \%) OF THE PROPOSED APPROACH COMPARED TO PREVIOUS WORK AND ALL-FEATURES CASE

\begin{tabular}{lcccccccc}
\hline Classification/Node & Waist & R-Wrist & L-Wrist & R-Arm & L-Thigh & R-Ankle & L-Ankle & Average \\
\hline kNN (all features) & 79 & 68 & 70 & 70 & 78 & 75 & 87 & 75.3 \\
SVM (all features) & 99.8 & 100 & 100 & 100 & 100 & 99.8 & 100 & 99.8 \\
ANN (selected features) & 99 & 100 & 100 & 100 & 98.2 & 93.5 & 100 & 98.7 \\
kNN (selected features) & 97 & 100 & 100 & 100 & 96 & 96 & 100 & 98.4 \\
RBF Network (selected features) & 99 & 99.8 & 98 & 100 & 87 & 82 & 100 & 95.1 \\
Compressive Sensing [6] & 95 & 99 & 98 & 99 & 92 & 92 & 98 & 96.1 \\
\hline
\end{tabular}

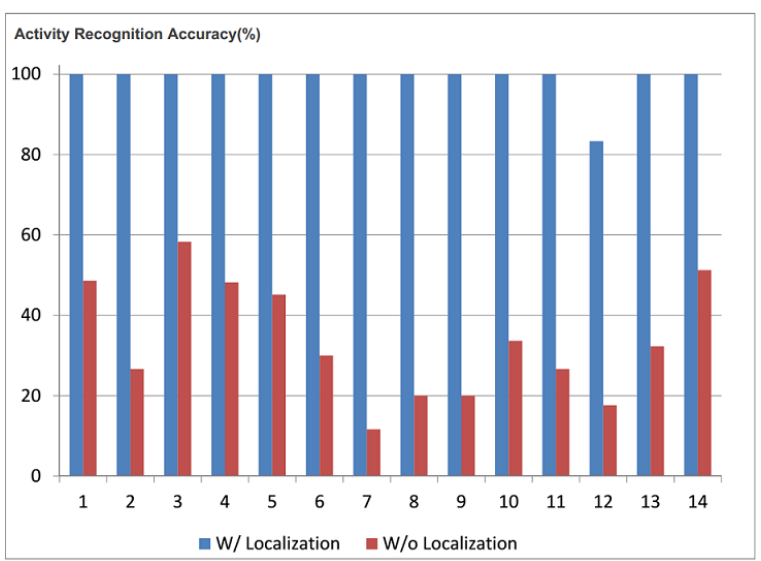

Fig. 4. Accuracy of activity recognition for individual movements with and without sensor localization

The accuracy of the activity recognition algorithm is reduced to an average of $33.6 \%$ in absence of a node localization algorithm.

\section{CONCLUSION}

In this paper, we presented an approach for on-body sensor location with applications in health and wellbeing. Our approach relies on computationally simple classification algorithms that operate on a small set of features extracted from wearable sensor nodes. We demonstrated the potential of this approach for accurate localization of wearable sensors. The algorithm not only outperforms previous techniques in terms of accuracy but also obtains much less computing complexity.

An important observation based on the results obtained in this paper is that a limited number of features extracted from a sensor node can be effectively used to precisely detect location of an on-body sensor. It is also interesting that these prominent features are associated with both accelerometer and gyroscope sensors.
A wearable sensor can be potentially worn on many different locations on the body. In this paper, we focused on an experimental setting with 7 body locations. In future, we plan on collecting data from larger body sensor networks where each body segment is associated with a wearable sensor. We plan on exploring inter-node correlations that neighboring nodes may exhibit which can potentially result in a hierarchical approach to sensor localization.

\section{REFERENCES}

[1] M. C. Riddell and R. J. Sigal, "Physical activity, exercise and diabetes." Canadian journal of diabetes, vol. 37, no. 6, pp. 359-360, 2013.

[2] A. J. Cooper, S. Brage, U. Ekelund, N. J. Wareham, S. J. Griffin, and R. K. Simmons, "Association between objectively assessed sedentary time and physical activity with metabolic risk factors among people with recently diagnosed type 2 diabetes," Diabetologia, vol. 57 , no. 1 , pp. 73-82, 2014.

[3] H. Ghasemzadeh, S. Ostadabbas, E. Guenterberg, and A. Pantelopoulos, "Wireless medical-embedded systems: A review of signalprocessing techniques for classification," Sensors Journal, IEEE, vol. 13, no. 2, pp. 423-437, Feb 2013.

[4] R. O. Duda, P. E. Hart, and D. G. Stork, Pattern Classification. WileyInterscience Publication, 2000.

[5] A. Vahdatpour, N. Amini, and M. Sarrafzadeh, "On-body device localization for health and medical monitoring applications," in Pervasive Computing and Communications (PerCom), 2011 IEEE International Conference on, March 2011, pp. 37-44.

[6] W. Xu, M. Zhang, A. Sawchuk, and M. Sarrafzadeh, "Co-recognition of human activity and sensor location via compressed sensing in wearable body sensor networks," in Wearable and Implantable Body Sensor Networks (BSN), 2012 Ninth International Conference on, May 2012, pp. 124-129.

[7] K. Kunze and P. Lukowicz, "Using acceleration signatures from everyday activities for on-body device location," in Wearable Computers, 2007 11th IEEE International Symposium on, Oct 2007, pp. 115-116.

[8] K. Kunze, P. Lukowicz, H. Junker, and G. Trster, "Where am i: Recognizing on-body positions of wearable sensors," in Location- and Context-Awareness, ser. Lecture Notes in Computer Science, T. Strang and C. Linnhoff-Popien, Eds. Springer Berlin Heidelberg, 2005, vol. 3479 , pp. 264-275.

[9] J. Lester, B. Hannaford, and G. Borriello, "are you with me?-using accelerometers to determine if two devices are carried by the same person," in Pervasive computing. Springer, 2004, pp. 33-50.

[10] M. A. Hall, "Correlation-based feature selection for machine learning," Ph.D. dissertation, The University of Waikato, 1999. 